\title{
Low-flow apnoeic oxygenation for laryngeal surgery: a prospective observational study
}

C. J. O'Loughlin ${ }^{1}$, D. J. Phyland², N. A. Vallance ${ }^{3}$, C. Giddings ${ }^{3}$, E. Malkoutzis ${ }^{4}$, E. Gunasekera ${ }^{1}$, A. Webb ${ }^{1}$, R. Barnes $^{4}$

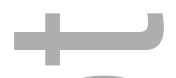

1 Consultant, Department of Anaesthesia, Frankston Private Hospital, Victoria, Australia

2 Associate Professor, 3 ENT Surgeon Department of Otolaryngology, Head and Neck Surgery, School of

Clinical Sciences (Monash Health), Monash University, Clayton, Victoria, Australia

4 Consultant, Department of Anaesthesia, Monash Health, Victoria, Australia

Correspondence to: R. Barnes

Email: richard.barnes@monashhealth.org

Keywords: apnoeic oxygenation; microlaryngoscopy; panendoscopy

Short title: Low-flow apnoeic oxygenation for laryngeal surgery

\section{Summary}

Laryngeal surgery requires a shared airway and close collaboration between surgeon and anaesthetist to optimise operating conditions. Apnoeic oxygenation uses the principle of aventilatory mass flow to maintain oxygenation of pulmonary capillary blood under apnoeic conditions while minimising laryngeal movement. Concerns regarding accumulation of carbon dioxide and resultant acidaemia have limited the use of the technique. We performed a prospective study of low-flow apnoeic oxygenation for patients having microlaryngoscopy under general anaesthesia in order to evaluate the ability of the technique to maintain oxygenation and determine the resultant rate of carbon dioxide accumulation. Sixty-four patients undergoing microlaryngoscopy under general anaesthesia were studied between November 2016 and December 2018. Intra-operative oxygenation was provided via a 10-French oxygen catheter placed into the trachea delivering oxygen at 0.5-1.0 I. $\mathrm{min}^{-1}$. Data regarding apnoea time, peripheral oxygen saturation and venous blood gas concentrations were recorded. The mean (SD)

This is the author manuscript accepted for publication and has undergone full peer review but has not been through the copyediting, typesetting, pagination and proofreading process, which may lead to differences between this version and the Version of Record. Please cite this article as doi: 10.1111/ANAE.14959

This article is protected by copyright. All rights reserved 
duration of apnoea was 18.7 (7.2) minutes. Apnoeic oxygenation allowed successful completion of the surgical procedure in 62/64 patients. Mean (SD) rate of rise of the venous partial pressure of carbon dioxide was $0.15(0.10) \mathrm{kPa} \cdot \mathrm{min}^{-1}$. Operating conditions were recorded qualitatively as being adequate in all cases. No adverse effects were reported. Low-flow intratracheal apnoeic oxygenation is a simple, effective and inexpensive technique to maintain oxygenation for laryngeal surgery.

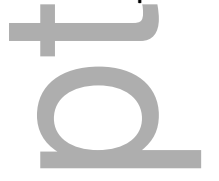

\section{Introduction}

Airway surgery presents a unique dynamic between the ear, nose and throat surgeon and anaesthetist; patient safety and anaesthetic requirements must be balanced with the need for adequate exposure of anatomical structures and optimal conditions for surgery [1,2]. There have been a number of anaesthetic techniques described to optimise conditions for airway surgery [2]. The ideal approach prioritises: patient safety and comfort; optimal surgical conditions (wide field of view and lack of movement of the larynx); uneventful postoperative recovery with day of surgery discharge where possible; and cost-effective use of resources. Optimal conditions are particularly important with the advent of microsurgical techniques used in phonosurgery, as both anaesthetist and surgeon share a very small operative field.

Apnoeic oxygenation is increasing in popularity as an alternative to traditional anaesthetic techniques [3]. Apnoeic oxygenation presents an opportunity to safely oxygenate the patient whilst keeping the airway free of obstruction and movement, and provide uninterrupted optimal surgical conditions for airway surgery. Apnoeic oxygenation using high-flow nasal oxygen (HFNO) has been studied in a variety of settings, in particular difficult airway management and airway surgery, and has been proposed to facilitate carbon dioxide clearance [4-7]. However, equipment to administer HFNO is not available universally, especially in settings of limited resources. In addition, HFNO carries a significant cost of consumables. An alternative technique to HFNO was described in 2013 by Rudlof and Hohenhorst, who found that apnoeic oxygenation with low-flow oxygen using an oxygen catheter inserted through the glottis into the trachea could be tolerated by patients for up to $45 \mathrm{~min}$ [8]. Of the 45 successful cases in their study, optimal operating conditions were described by all surgeons (mean operating time $25 \mathrm{~min}$ ). However, the authors did not report any blood gas analyses in their data, meaning that the efficacy of the technique for carbon dioxide clearance remains unclear.

Considering recent interest in apnoeic oxygenation and HFNO, and having used the technique adapted This article is protected by copyright. All rights reserved 
from Rudlof and colleagues in our institution for a number of years, we undertook a prospective observational study of low-flow intra-tracheal apnoeic oxygenation for microlaryngeal surgery. We aimed to confirm the ability of the technique to maintain oxygenation and quantify the resultant venous carbon dioxide accumulation.

\section{Methods}

This prospective observational study was approved by the Monash Health Human Research Ethics Committee and the hospital executive at Frankston Private Hospital, Frankston, Victoria, Australia. All patients gave written consent pre-operatively in accordance with standard ethical guidelines.

All adult patients (aged > 18 years) due to undergo microlaryngoscopy for microlaryngeal surgery (e.g. vocal fold augmentation, biopsy, phonosurgery or subglottic stenosis dilatation) with either of the two study laryngologists were considered for inclusion. Exclusion criteria were: BMI > 45; any condition or effect of a condition that might be exacerbated by hypercapnia (e.g. neurological space-occupying lesion, pulmonary hypertension); severe cardiorespiratory disease; and predicted prolonged surgery.

Minimum intra-operative monitoring following the guidelines set by the Australian and New Zealand College of Anaesthetists was applied, with additional monitoring at the discretion of the treating anaesthetist. Following pre-oxygenation with 100\% oxygen for $3 \mathrm{~min}$, anaesthesia was induced using propofol-based total intravenous anaesthesia along with an opioid and neuromuscular blocking agent (specific drugs and doses at the discretion of the treating anaesthetist). A 10-French oxygen catheter (Thermo Fisher Scientific, Scoresby, Victoria, Australia) was then placed in the trachea using direct laryngoscopy (Fig. 1). The patients' lungs were then ventilated using $100 \%$ oxygen (either via facemask or supraglottic airway device) aiming to achieve an end-tidal oxygen concentration $>90 \%$ until the surgeon was ready to commence. Following insertion of the suspension laryngoscope, apnoeic oxygenation was commenced via the oxygen cannula at a flow rate of 0.5-1.0 I. $\mathrm{min}^{-1}$ (depending on the flowmeter available). Upon completion of the surgical procedure and removal of the operative laryngoscope, the oxygen catheter was removed and a supraglottic airway device inserted. Neuromuscular blockade was then antagonised (specific agents at discretion of the treating anaesthetist) and spontaneous ventilation established.

In order to measure the venous partial pressure of carbon dioxide $\left(\mathrm{P}_{\mathrm{v}} \mathrm{CO}_{2}\right)$, venous blood samples were 
collected from the intravenous cannula at the following points: time of cannula insertion (baseline); cessation of ventilation (start of apnoea); and completion of the procedure prior to commencement of ventilation (end of apnoea). Other data collected included: oxygen saturations at the same three time points as venous blood sampling; duration of time with peripheral capillary oxygen saturation $\left(\mathrm{SpO}_{2}\right)<$ 95\%; duration of time with $\mathrm{SpO}_{2}<92 \%$; whether the technique was abandoned and reason for abandonment; overall duration of surgery; and surgical view (adequate or inadequate). Partway through the study the protocol was amended to also collect data on end-tidal carbon dioxide levels at commencement and end of apnoea for comparison with venous values. Criteria for abandonment of the technique included: inadequate oxygenation; spontaneous ventilation occurring during surgery; and/or any other concerns regarding patient safety (e.g. signs of myocardial ischaemia, significant arrhythmia or haemodynamic instability). Data were collated and analysed using Microsoft Office Excel (Microsoft Corporation; Redmond, WA, USA).

\section{Results}

During the period from November 2016 to December 2018, we recruited 66 patients. Two cases were excluded from analysis due to deviation from the protocol. In one case, the patient had an initial Cormack-Lehane grade 4 view at laryngoscopy due to a base of tongue tumour, and the anaesthetist made the decision to abandon the technique and secure the airway using videolaryngoscopy and a microlaryngeal tube. In another case, the technique was abandoned due to operator error, where desaturation occurred due to failure to re-initiate oxygen flow after it was turned off to allow balloon dilatation of subglottic stenosis, requiring jet ventilation to restore oxygenation. The final analysis therefore included 64 patients (42 female), mean (SD) age 47 (16) years and mean (SD) BMI 25 (4) kg.m 2. Thirty-two patients were graded ASA physical status grade 1, 26 were grade 2 and six patients were grade 3. All patients underwent laryngeal surgery for exploratory microlarygoscopy or phonosurgery.

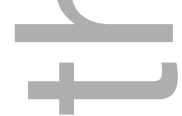

All patients had $\mathrm{SpO}_{2} 98 \%-100 \%$ at commencement of apnoea. Apnoeic oxygenation allowed successful completion of the surgical procedure in 62/64 cases (Fig. 2). In the 62 successful cases, mean (SD) duration of apnoeic oxygenation (and surgery) was 18.7 (7.2) $\mathrm{min}$. In all these cases the view was recorded as adequate by the operating surgeon and in no cases was the technique abandoned due to inadequate view. Of the successful cases, 58 were completed without significant oxygen desaturation documented (including seven cases where end-apnoea oxygen saturation data were not recorded, though no desaturation or technique termination was reported). Three of the successful cases had mild desaturation $\left(\mathrm{SpO}_{2} 92-95 \%\right)$ allowing completion of the procedure: one patient was asthmatic and This article is protected by copyright. All rights reserved 
complained of wheeze in the post-anaesthetic care unit requiring nebulised salbutamol; one patient was an ex-smoker being investigated for chronic cough; and one patient desaturated to $\mathrm{SpO}_{2} 87 \%$ but only upon completion of the procedure, as the suspension laryngoscope was being removed. In 2/64 cases, the technique was abandoned due to desaturation (after $9 \mathrm{~min}$ and $11 \mathrm{~min}$ respectively): one patient was asthmatic, although there was no evidence of bronchospasm peri-operatively; the second was an older patient (71 years) who was a smoker. No cases were abandoned due to signs of myocardial ischaemia, arrhythmia or haemodynamic instability. Oxygenation was recovered easily in all patients either via facemask ventilation, supraglottic airway device insertion or low frequency jet ventilation to facilitate conclusion of the surgical procedure.

Data relating to $\mathrm{P}_{\mathrm{v}} \mathrm{CO}_{2}$ were obtained for all 62 cases where the procedure was completed using the apnoeic technique (Fig. 3). The mean (SD) rise in $\mathrm{P}_{\mathrm{v}} \mathrm{CO}_{2}$ was $2.60(1.67) \mathrm{kPa}$ and mean (SD) rate of rise was $0.15(0.10) \mathrm{kPa} \cdot \mathrm{min}^{-1}$. End-tidal carbon dioxide data were recorded for 28 patients, all of whom completed the surgical procedure using the apnoeic oxygenation technique. The mean (SD) rise of endtidal carbon dioxide was 2.19 (1.29) kPa and mean (SD) rate of rise was 0.12 (0.07) kPa.min ${ }^{-1}$.

\section{Discussion}

Maintenance of oxygenation during apnoea by aventilatory mass flow has been achieved using a variety of techniques, with particular utility in microlaryngeal surgery and difficult airway management [4-8]. This observational study shows that low-flow tracheal apnoeic oxygenation using simple, readily available equipment can be used for short- to medium-duration laryngeal procedures, providing adequate operating conditions. In our study, low-flow apnoeic oxygenation with a narrow-bore transglottic catheter allowed successful completion of the microlaryngoscopy procedure in 62/64 cases when employed as per our protocol. Two patients desaturated to $\mathrm{SpO}_{2} 92 \%$ resulting in abandonment of the technique, and one further patient desaturated to $\mathrm{SpO}_{2}<92 \%$ only after the procedure was completed. In most cases where there was significant oxygen desaturation, an identifiable risk factor such as smoking or asthma was present. These results are similar to those of Rudlof et al., who maintained adequate oxygenation for a mean operating period of $25 \mathrm{~min}$ in 44/47 patients, using the same technique [8].

These results regarding oxygenation are also comparable to those found with apnoeic oxygenation using HFNO. Patel et al. used HFNO for maintenance of oxygenation during securement of difficult airways, with a median apnoea time of $14 \mathrm{~min}$ [4]. Of the 25 cases reported, four reached $\mathrm{SpO}_{2}$ nadirs of $\leq 92 \%$ This article is protected by copyright. All rights reserved 
(the cut-off level we set in our study), though the median BMI was $30 \mathrm{~kg} \cdot \mathrm{m}^{-2}$ and nine patients had acute airway compromise, likely increasing the likelihood of desaturation. In Lyons and Callaghan's study of HFNO in microlaryngeal surgery with a median apnoea time of $19 \mathrm{~min}, 4 / 28$ patients desaturated to $\mathrm{SpO}_{2}<90 \%$ [6]. In a study by Gustaffson et al. using HFNO for airway surgery, 2/31 patients desaturated to $\mathrm{SpO}_{2} 92 \%$ over a mean apnoea time of $22.5 \mathrm{~min}$ [5].

Historically, the primary limitation of apnoeic oxygenation has been concerns regarding carbon dioxide production and accumulation, with resultant acidaemia and its deleterious physiological effects. Studies of apnoeic oxygenation have shown the overall rate of increase of carbon dioxide concentration to be 0.15-0.45 kPa.min-1; this increase occurs in a non-linear fashion (Table 1). Frumin et al. showed a rise in arterial partial pressure of carbon dioxide $\left(\mathrm{P}_{\mathrm{a}} \mathrm{CO}_{2}\right)$ of $0.4 \mathrm{kPa} \cdot \mathrm{min}^{-1}$ in eight apnoeic subjects either during or after surgery lasting 18-55 min [9]. Similarly, Fraioli et al. showed a mean increase in $\mathrm{P}_{\mathrm{a}} \mathrm{CO}_{2}$ of 0.43 $\mathrm{kPa} \cdot \mathrm{min}^{-1}$ in 31 patients over $15 \mathrm{~min}$ of apnoea [10]. Eger and Severinghaus showed a rise in end-tidal carbon dioxide of $1.8 \mathrm{kPa}$ in the first minute and $0.4 \mathrm{kPa} \cdot \mathrm{min}^{-1}$ thereafter in five anaesthetised patients, with an overall mean rise of $0.56 \mathrm{kPa} \cdot \mathrm{min}^{-1}$ [11]. Stock et al. showed a similar mean rate of rise of $\mathrm{P}_{\mathrm{a}} \mathrm{CO}_{2}$ of $1.6 \mathrm{kPa}$ in the first minute and $0.45 \mathrm{kPa} \cdot \mathrm{min}^{-1}$ thereafter, when tracheal tubes were clamped in anaesthetised patients for up to $5 \min [12]$.

Based on this evidence, it has been stated that low-flow oxygenation during apnoea does not significantly affect carbon dioxide clearance $[4,6,10]$. However, Rudlof et al. showed a mean rise in endtidal carbon dioxide of $0.24 \mathrm{kPa} \cdot \mathrm{min}^{-1}$ over a mean apnoea time of 25 min using low-flow intratracheal apnoeic oxygenation [8]; this is higher than that observed in our study that used the same technique (rate of increase in $\mathrm{P}_{\mathrm{V}} \mathrm{CO}_{2}$ of $0.15 \mathrm{kPa} \cdot \mathrm{min}^{-1}$ and mean increase in end-tidal carbon dioxide of 0.12 $\left.\mathrm{kPa} \cdot \mathrm{min}^{-1}\right)$. High-flow nasal oxygen techniques have claimed to produce significant carbon dioxide clearance and thus provide a degree of ventilation as well as oxygenation. Patel et al. reported an increase in end-tidal carbon dioxide of $0.15 \mathrm{kpa} \cdot \mathrm{min}^{-1}$ [4], whilst Lyons and Callaghan reported a rate of rise of peripheral $\mathrm{P}_{\mathrm{V}} \mathrm{CO}_{2}$ of $0.21 \mathrm{kPa} \cdot \mathrm{min}^{-1}$ over the initial $15 \mathrm{~min}$ of apnoea [6]. The latter study concluded that HFNO during apnoea provides approximately $50 \%$ of 'optimal ventilation' with respect to increase in $\mathrm{P}_{\mathrm{v}} \mathrm{CO}_{2}$.

The study by Gustaffson et al. helps to clarify these competing claims [5]. They showed a similarly slow rise in end-tidal carbon dioxide (0.12 kPa. $\mathrm{min}^{-1}$ over $22.5 \mathrm{~min}$ of apnoea.) but a much faster rise in $\mathrm{P}_{\mathrm{a}} \mathrm{CO}_{2}$ of $0.24 \mathrm{kPa}$. $\mathrm{min}^{-1}$ with a concordant fall in $\mathrm{pH}$. This illustrates the increased alveolar-arterial gradient for This article is protected by copyright. All rights reserved 
carbon dioxide and the unreliability of end-tidal carbon dioxide measurements after significant apnoea. In addition, Toner et al. monitored transcutaneous carbon dioxide concentration $\left(\mathrm{P}_{\mathrm{tc}} \mathrm{CO}_{2}\right)$ during apnoeic oxygenation with oxygen flows of $10 \mathrm{I} \cdot \mathrm{min}^{-1}$ over $12.5 \mathrm{~min}$ of apnoea, and found a rate of rise of 0.30 $\mathrm{kPa} \cdot \mathrm{min}^{-1}[13]$.

From the literature it therefore appears that peripheral venous and end-tidal carbon dioxide measurements may significantly underestimate carbon dioxide accumulation, and that future studies should utilise arterial or its surrogate, transcutaneous carbon dioxide measurement. While peripheral venous $\mathrm{pH}$ correlates well with arterial $\mathrm{pH}, \mathrm{P}_{\mathrm{v}} \mathrm{CO}_{2}$ values show more variability [14]. During apnoea, $\mathrm{P}_{\mathrm{v}} \mathrm{CO}_{2}$ initially equilibrates with $\mathrm{P}_{\mathrm{a}} \mathrm{CO}_{2}$; subsequently $\mathrm{P}_{\mathrm{a}} \mathrm{CO}_{2}$ exceeds $\mathrm{P}_{\mathrm{v}} \mathrm{CO}_{2}$, due to retention of carbon dioxide in the pulmonary circulation as well as the Haldane effect [3]. Measurements of $\mathrm{P}_{\mathrm{tc}} \mathrm{CO}_{2}$ correlate well with $\mathrm{P}_{2} \mathrm{CO}_{2}$, and could be used in future similar studies to assess more accurately the degree of carbon dioxide accumulation and respiratory acidosis developing during apnoea in a continuous and non-invasive fashion [5]. Whether HFNO techniques significantly increase carbon dioxide clearance remains unclear, and has been proposed as the subject of future randomised control trials [15]. It is also noteworthy that historical studies showed significantly higher rates of carbon dioxide rise during apnoea than more recent evidence. This could be due to differences in metabolic rate with modern anaesthetic techniques compared to older practices, such as repeated doses of succinylcholine, which has been shown to increase carbon dioxide production [16].

There are a number of limitations to this study worthy of consideration. First, the study was observational in nature and performed in two centres. Second, most patients in our study were not obese and ASA physical status 1-2; our low-flow apnoeic oxygenation technique may be unsuitable for obese patients or those with significant cardiorespiratory co-morbidities. Third, we did not utilise a validated scoring system to grade surgical exposure. However, in our experience the technique provides excellent operating conditions for microlaryngoscopy with complete akinesia and good access to vocal folds for the treatment of laryngeal pathology. The small mobile catheter is easily manipulated allowing exposure of the posterior glottis and causes minimal surgical interference although lesions of the posterior glottis may still be obscured by the small bore catheter or tube, and may require true tubeless anaesthesia. Fourth, due to ethical constraints, $\mathrm{P}_{\mathrm{v}} \mathrm{CO}_{2}$ was used in our study as a measure of carbon dioxide accumulation, but, as discussed earlier, this is only an approximation of $\mathrm{P}_{\mathrm{a}} \mathrm{CO}_{2}$. Furthermore, carbon dioxide accumulation has been shown to be non-linear with an initial rapid rise followed by a plateau phase [5,11-13]. The mean rate of rise will therefore depend on the apnoea time [15]. Blood 
samples were taken only at the start and end of apnoea, and we therefore cannot comment on the linearity of carbon dioxide rise in our study population. Finally, while we attempted to ensure complete apnoea with neuromuscular blockade, it is possible that patients may re-establish diaphragm movement and small volume ventilation as carbon dioxide levels rise and neuromuscular blockade wears off. With a low-flow technique, room air may be entrained as a result, thereby reducing the effectiveness of apnoeic oxygenation and potentially leading to premature desaturation [8]. Neuromuscular monitoring and/or high-dose neuromuscular blocking agent techniques are potential strategies to attenuate this risk.

In conclusion, the data from this observational study supports the use of low-flow tracheal apnoeic oxygenation with a narrow-bore catheter to oxygenate non-obese patients for short duration laryngeal surgery, providing adequate operating conditions and without excessive accumulation of carbon dioxide.

\section{Acknowledgements and competing interests}

No external funding or competing interests declared.

\section{References}

1. Weissberger EC, Emhardt JD. Apneic anesthesia with intermittent ventilation for microsurgery of the upper airway. Laryngoscope 1996; 106: 1099-1102.

2. Pearson KL, McGuire BE. Anaesthesia for laryngo-tracheal surgery, including tubeless field techniques. BJA Education 2017; 17(7): 242-8

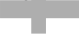

3. Lyons $\mathrm{C}$, Callaghan $\mathrm{M}$. Uses and mechanisms of apnoeic oxygenation: a narrative review. Anaesthesia 2019; 74: 497-507

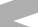

4. Patel A, Nouraei SA. Transnasal Humidified Rapid-Insufflation Ventilatory Exchange (THRIVE): a physiological method of increasing apnoea time in patients with difficult airways. Anaesthesia 2015; 70: $323-9$ 
5. Gustafsson IM, Lodenius A, Tunelli J, Ullman J, Jonsson-Fagerlund M. Apnoeic oxygenation in adults under general anaesthesia using transnasal humidified rapid-insufflation ventilatory exchange (THRIVE) - a physiological study. British Journal of Anaesthesia 2017; 118: 610-7

6. Lyons C, Callaghan M. Apnoeic oxygenation with high-flow nasal oxygen for laryngeal surgery: a case series. Anaesthesia 2017; 72: 1379-87

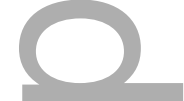

7. Ebeling CG, Riccio CA. Apneic oxygenation with high-flow nasal cannula and transcutaneous carbon dioxide monitoring during airway surgery: a case series. Anesthesia and Analgesia 2019; 12(10): 3668

8. Rudlof B, Hohenhorst W. Use of apneic oxygenation for the performance of pan-endoscopy. Otolaryngology-Head and Neck Surgery 2013; 149(2): 235-9

9. Frumin MJ, Epstein RM, Cohen G. Apneic oxygenation in man. Anesthesiology 1959; 20(6):789-798

10. Fraioli RL, Sheffer LA, Steffenson JL. Pulmonary and cardiovascular effects of apneic oxygenation in man. Anesthesiology 1973; 39: 588-96

11. Eger El, Severinghaus JW. The rate of rise of $\mathrm{PaCO}_{2}$ in the apneic anesthetized patient. Anesthesiology 1961; 22: 419-25

12. Stock MC, Schisler JQ, McSweeney TD. The PaCO2 rate of rise in anesthetized patients with airway obstruction. Journal of Clinical Anesthesia 1989; 1: 328-32

13. Toner AJ, Douglas SG, Bailey MA, Avis HJ, Pillai AV, Phillips M, Heard A. Effect of apneic oxygenation on tracheal oxygen levels, tracheal pressure, and carbon dioxide accumulation: a randomized, controlled trial of buccal oxygen administration. Anesthesia and Analgesia 2019; 128(6) 1154-9

14. Byrne AL, Bennett M, Chatterji R, Symons R, Pace NL, Thomas PS. Peripheral venous and arterial blood gas analysis in adults: are they comparable? A systematic review and meta-analysis. Respirology 2014; 19(2):168

15. Toner AJ. Carbon dioxide clearance during apnoea with high-flow nasal oxygen: epiphenomenon or This article is protected by copyright. All rights reserved 
a failure to THRIVE? Anaesthesia 2019. Epub ahead of print 6 September.

https://doi.org/10.1111/anae.14848

16. Christensen KJ, Olesen AS, Jorgensen S. Effects of suxamethonium on CO2-production. Acta Anaesthesiologica Scandinavica 1985; 29: 424-6.

Table 1. Reported rates of carbon dioxide $\left(\mathrm{CO}_{2}\right)$ accumulation under different apnoeic conditions.

\begin{tabular}{lll} 
& & \\
\hline Study & Method of $\mathrm{CO}_{2}$ detection & Rate of rise; kPa.min ${ }^{-1}$ \\
\hline Frumin et al. [9] & Arterial & 0.40 \\
Fraioli et al. [10] & Arterial & 0.43 \\
Eger and Severinghaus [11] & End-tidal & 0.40 \\
Stock et al. [12] & Arterial & 0.45 \\
Rudlof and Hohenhurst [8] & End-tidal & 0.24 \\
Patel and Nouraei [4] & End-tidal & 0.15 \\
Gustaffson et al. [5] & Arterial & 0.24 \\
\hline Lyons and Callaghan [3] & & 0.21 \\
\hline Toner et al. [13] & Venous & 0.30 \\
\hline
\end{tabular}

Figure 1. Microlaryngoscopy view showing a 10-French oxygen catheter in-situ. 
Figure 2. Oxygen saturation after the use of a low-flow apnoeic oxygenation technique in patients having laryngeal surgery $(n=57)$. Overlapping data have been stacked for display. Hollow diamonds represent the two cases where the technique was abandoned due to desaturation.

Figure 3. Venous partial pressure of carbon dioxide $\left(\mathrm{P}_{\mathrm{v}} \mathrm{CO}_{2}\right)$ after the use of a low-flow apnoeic oxygenation technique in patients having laryngeal surgery $(n=62)$. The dashed line represents the mean $\mathrm{P}_{\mathrm{v}} \mathrm{CO}_{2}(6.09 \mathrm{kPa})$ at the start of apnoea for comparison.

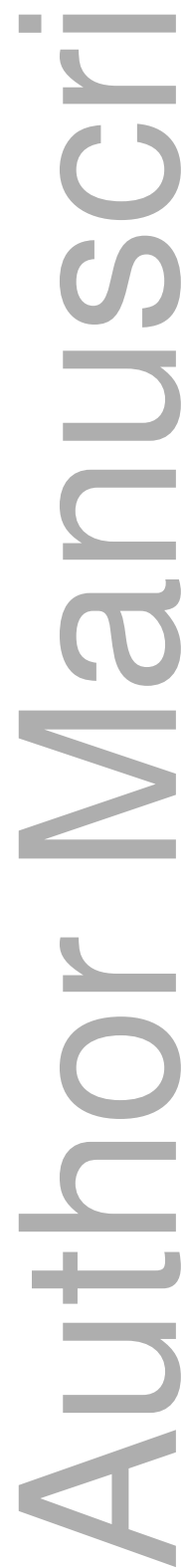

This article is protected by copyright. All rights reserved 

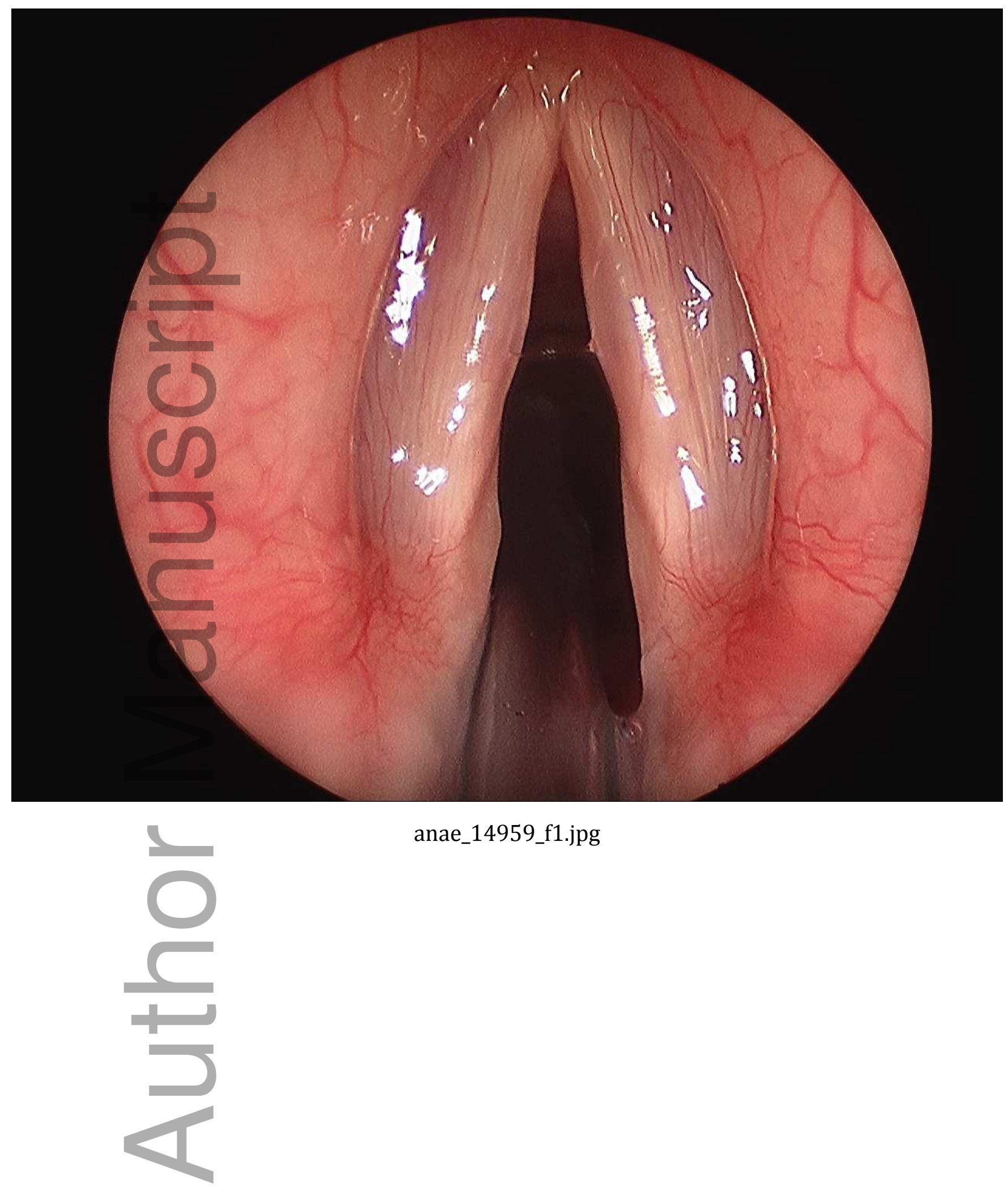

anae_14959_f1.jpg

This article is protected by copyright. All rights reserved 


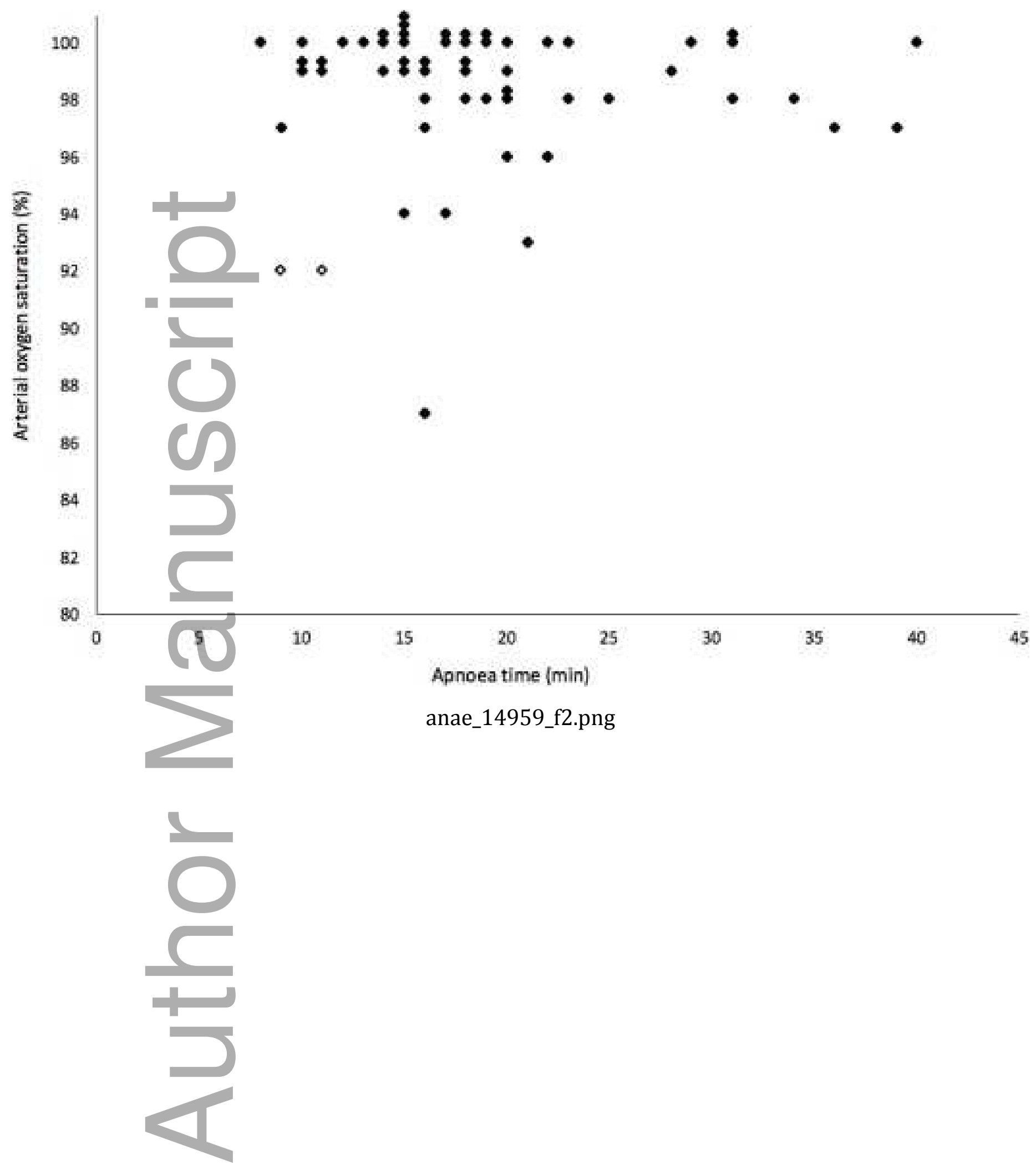

This article is protected by copyright. All rights reserved 


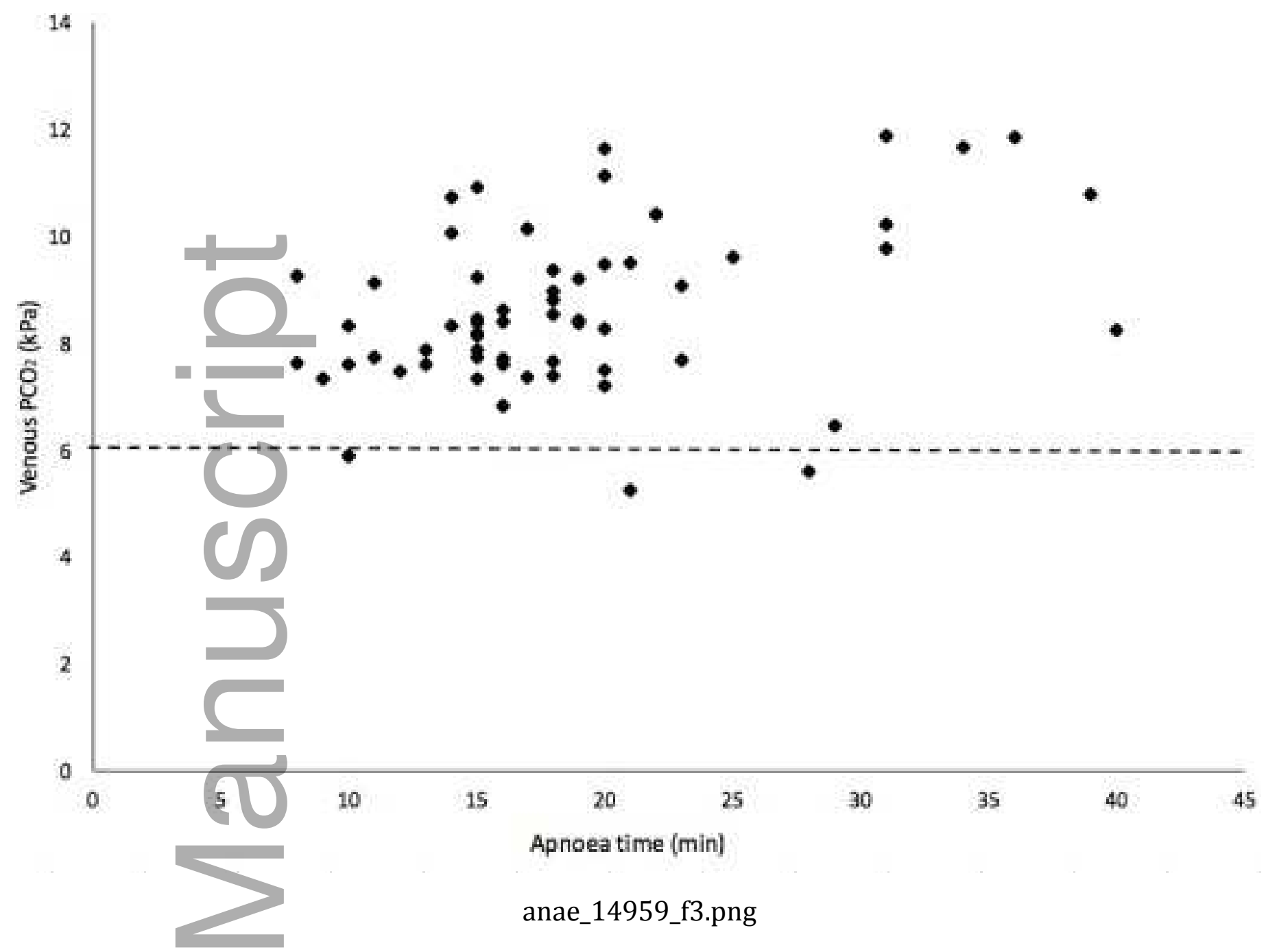

This article is protected by copyright. All rights reserved 


\section{University Library}

\section{- M M N E R VA A gateway to Melbourne's research publications}

Minerva Access is the Institutional Repository of The University of Melbourne

Author/s:

O'Loughlin, CJ;Phyland, DJ;Vallance, NA;Giddings, C;Malkoutzis, E;Gunasekera, E;Webb, A;Barnes, $R$

Title:

Low-flow apnoeic oxygenation for laryngeal surgery: a prospective observational study

Date:

2019-12-23

Citation:

O'Loughlin, C. J., Phyland, D. J., Vallance, N. A., Giddings, C., Malkoutzis, E., Gunasekera, E., Webb, A. \& Barnes, R. (2019). Low-flow apnoeic oxygenation for laryngeal surgery:

a prospective observational study. ANAESTHESIA, 75 (8), pp.1070-1075. https:// doi.org/10.1111/anae.14959.

Persistent Link:

http://hdl.handle.net/11343/286778 